\title{
On packing spanning arborescences with matroid constraint
}

\author{
Quentin Fortier a,1 Csaba Király b,2 Zoltán Szigeti a,3 \\ Shin-ichi Tanigawa ${ }^{\mathrm{c}, 4}$
}

a Univ. Grenoble Alpes, G-SCOP, Grenoble, France

b ELTE Eötvös Loránd University, Budapest, Hungary

c RIMS, Kyoto University, Sakyo-ku, Kyoto, Japan and

Centrum Wiskunde 83 Informatica (CWI),Amsterdam, The Netherlands

\begin{abstract}
Let us be given a rooted digraph $D=(V+s, A)$ with a designated root vertex $s$. Edmonds' seminal result [3] states that $D$ has a packing of $k$ spanning $s$-arborescences if and only if $D$ has a packing of $k(s, t)$-paths for all $t \in V$, where a packing means arc-disjoint subgraphs.

Let $\mathcal{M}$ be a matroid on the set of arcs leaving $s$. A packing of $(s, t)$-paths is called $\mathcal{M}$-based if their arcs leaving $s$ form a base of $\mathcal{M}$ while a packing of $s$ arborescences is called $\mathcal{M}$-based if, for all $t \in V$, the packing of $(s, t)$-paths provided by the arborescences is $\mathcal{M}$-based. Durand de Gevigney, Nguyen and Szigeti proved in [2] that $D$ has an $\mathcal{M}$-based packing of $s$-arborescences if and only if $D$ has an $\mathcal{M}$-based packing of $(s, t)$-paths for all $t \in V$. Bérczi and Frank conjectured that this statement can be strengthened in the sense of Edmonds' theorem such that each $s$-arborescence is required to be spanning. Specifically, they conjectured that $D$ has an $\mathcal{M}$-based packing of spanning $s$-arborescences if and only if $D$ has an $\mathcal{M}$-based packing of $(s, t)$-paths for all $t \in V$.

We disprove this conjecture in its general form and we prove that the corresponding decision problem is NP-complete. However, we prove that the conjecture
\end{abstract}


holds for several fundamental classes of matroids, such as graphic matroids and transversal matroids.

Keywords: connectivity, packing arborescences, Edmonds' branching theorem, matroid

\section{Introduction}

Packing arborescences, or more generally, packing problems concerning connectivity in directed graphs are fundamental subjects in combinatorial optimization. Here, by packing subgraphs in a directed graph, we mean a set of arc-disjoint subgraphs. The question of reachability is one of the basics in the area of connectivity in digraphs. Suppose that we are given a rooted digraph, i.e. a digraph $D=(V+s, A)$ with a designated root vertex $s$. Let $S$ be the set of vertices reachable from $s$ in $D$. The definition of the reachability says that, for each $t \in S, D$ has an $(s, t)$-path, which certificates that $t$ belongs indeed to $S$. Now, consider storing such certificates for all vertices in $S$. Then storing an $s$-arborescence on $S$ would be the most compact way for keeping all the certificates simultaneously.

To extend this idea to a more general setting, suppose that $D$ has a packing of $k(s, t)$-paths from $s$ to each vertex $t$ in $V$, and suppose that we want to provide a certificate that $D$ has indeed such a property. Then the most compact certificate would be to exhibit $k$ arc-disjoint spanning $s$-arborescences in $D$. The following fundamental theorem of Edmonds [3] claims that such a compact certificate always exists.

Theorem 1.1 ([3]) There exists a packing of $k$ spanning s-arborescences in a rooted digraph $D=(V+s, A)$ if and only if there exists a packing of $k$ $(s, t)$-paths in $D$ for every $t \in V$.

The problem of packing $k(s, t)$-paths is equivalent to asking whether one can send $k$ distinct commodities from $s$ to $t$ by assuming that each arc can transmit at most one commodity. Then what happens if commodities have a more involved independence structure? Here we are interested in a situation that each arc from the root can be used to transmit only a particular commodity, and we would like to know whether every vertex can receive a sufficient

$\overline{1 \text { Email: }}$ quentin.fortier@grenoble-inp.fr

2 Email: cskiraly@cs.elte.hu

3 Email: zoltan.szigeti@grenoble-inp.fr

4 Email: tanigawa@kurims.kyoto-u.ac.jp 
amount of independent commodities to understand the whole structure.

More formally, suppose that we are given a matroid-rooted digraph $(D=(V+s, A), \mathcal{M})$, i.e. a matroid $\mathcal{M}$ is given on the set of arcs leaving the root $s$ that we call root arcs. We are interested in a packing of $(s, t)$-paths whose root arcs form a base of $\mathcal{M}$. Such a packing is said to be an $\mathcal{M}$-based packing of $(s, t)$-paths. A packing of $s$-arborescences is called $\mathcal{M}$-based if, for all $t \in V$, the packing of $(s, t)$-paths provided by the arborescences is $\mathcal{M}$ based. A natural question is whether Edmonds' theorem can be extended for $\mathcal{M}$-based packings. A result of Durand de Gevigney, Nguyen and Szigeti [2] gives a partial answer to this question by showing the equivalence of the existence of an $\mathcal{M}$-based packing of s-arborescences in $D$ and an $\mathcal{M}$-based packing of $(s, t)$-paths in $D$ for every $t \in V$.

Notice that at the quantitative level, Theorem 1.1 always guarantees the existence of $k$ spanning $s$-arborescences while the number of $s$-arborescences in the result of [2] may be more than the rank of $\mathcal{M}$ since these arborescences are not necessarily spanning. K. Bérczi and A. Frank [5] conjectured that the result of [2] can be strengthened in the sense of Edmonds' theorem. This conjecture appeared also in a paper of Bérczi, T. Király and Kobayashi [1]. More formally, the conjecture is the following.

Conjecture $1.2([1])$ Let $(D=(V+s, A), \mathcal{M})$ be a matroid-rooted digraph. There exists an $\mathcal{M}$-based packing of spanning s-arborescences in $D$ if and only if there exists an $\mathcal{M}$-based packing of $(s, t)$-paths in $D$ for every $t \in V$.

We note that in [2], the result was proved in a slightly stronger form as stated in our introduction by imposing an extra technical condition as follows. Let $(D=(V+s, A), \mathcal{M})$ be a matroid-rooted digraph. $D$ is called $\mathcal{M}$-independent if the arc set leaving $s$ and entering $v$ is independent in $\mathcal{M}$ for every $v \in V$. This condition ensures that all root arcs can be used in an $\mathcal{M}$-based packing of $s$-arborescences in $D$. We will also use this property in some of our results.

Definitions. An $s$-arborescence is a directed tree on a vertex-set containing the root vertex $s$ in which each vertex has in-degree 1 except $s$. An $s$-arborescence in a digraph $D=(V+s, A)$ is spanning if its vertex set is $V+s$. For an $s$-arborescence $T$ and a vertex $v \neq s$ of $T$, we denote the unique arc of $T$ entering $v$ by $\boldsymbol{T}(\boldsymbol{v})$. For disjoint sets $X, Y \subseteq V+s$, we denote by $\partial_{\boldsymbol{X}}^{D}(\boldsymbol{Y})$ the subset of arcs in $D$ with tail in $X$ and head in $Y$. The superscript $D$ will be omitted, when it is clear from the context. The in-degree of a set $X \subseteq V+s$ is denoted by $\varrho_{\boldsymbol{D}}(\boldsymbol{X}):=\left|\partial_{V+s-X}^{D}(X)\right|$.

We will use standard terminology from matroid theory, such as rank func- 
tions, independent sets, and bases. For details, we refer to [7]. We usually denote a matroid $\mathcal{M}$ by a pair $(\mathrm{S}, r)$ of the ground set $\mathrm{S}$ and the rank function $r: 2^{\mathrm{S}} \rightarrow \mathbb{Z}$. Two elements $\mathrm{a}, \mathrm{a}^{\prime} \in \mathrm{S}$ are said to be parallel in $\mathcal{M}=(\mathrm{S}, r)$ (in notation, $\left.\mathbf{a} \| \mathbf{a}^{\prime}\right)$ if $r(\{\mathrm{a}\})=r\left(\left\{\mathbf{a}^{\prime}\right\}\right)=r\left(\left\{\mathrm{a}, \mathrm{a}^{\prime}\right\}\right)=1$.

We say that a matroid-rooted digraph $\left(D=(V+s, A), \mathcal{M}=\left(\partial_{s}(V), r\right)\right)$ is rooted $\mathcal{M}$-arc-connected $(\mathcal{M}$-ac for short) if there exists an $\mathcal{M}$-based packing of $(s, t)$-paths for all vertices $t$ in $V$. One can easily prove a Mengertype theorem saying that $D$ is rooted $\mathcal{M}$-arc-connected if and only if

$$
r\left(\partial_{s}(X)\right)+\varrho_{D-s}(X) \geq r(\mathcal{M}) \text { for all } X \subseteq V,
$$

where $\boldsymbol{r}(\mathcal{M})$ denotes the rank of $\mathcal{M}$. For simplicity, we will call an $\mathcal{M}$ based packing of spanning $s$-arborescences in $D$ that covers $\partial_{s}(V)$ a feasible packing.

The following classes of matroids will be discussed in this paper. Given a graph $G=(V, E)$ with a bijection $\pi: E \rightarrow \mathrm{S}$, a matroid on $\mathrm{S}$ with independent sets in $\mathcal{I}:=\{\pi(F): F$ is the edge set of a forest of $G\}$ is called a graphic matroid. A Fano matroid is a rank-3 matroid derived from the Fano plane (the smallest projective plane with 7 points) on a 7-element ground set (the points of the Fano plane) where every set of cardinality 3 is a base except the lines of the Fano plane. Given a bipartite graph $G=(S, T ; E)$ with a bijection $\pi: S \rightarrow \mathrm{S}$, a matroid on $\mathrm{S}$ with independent sets in $\mathcal{I}:=\{\pi(X): X \subseteq S$ that can be covered by a matching in $G\}$ is called a transversal matroid. It is well-known that a graphic matroid is always representable by a connected graph and a transversal matroid is always representable by a bipartite graph where $|T|$ is equal to the rank. It is also well-known that a matroid of rank at most 3 is not graphic if and only if it has a "minor" isomorphic to the Fano matroid or $U_{2,4}$.

\section{Results}

We prove that Conjecture 1.2 is true for several fundamental classes of matroids such as graphic and transversal matroids.

Theorem 2.1 Let $\left(D=(V+s, A), \mathcal{M}=\left(\partial_{s}(V), r\right)\right)$ be a matroid-rooted digraph with $r(\mathcal{M}) \leq 2$. There exists an $\mathcal{M}$-based packing of spanning $s$ arborescences in $D$ that covers $\partial_{s}(V)$ if and only if $D$ is $\mathcal{M}$-independent and $\mathcal{M}-a c$.

Theorem 2.2 Let $(D=(V+s, A), \mathcal{M})$ be a matroid-rooted digraph where $\mathcal{M}=\left(\partial_{s}(V), r\right)$ is a graphic matroid. There exists an $\mathcal{M}$-based packing of spanning s-arborescences in $D$ covering $\partial_{s}(V)$ if and only if $D$ is $\mathcal{M}$-ac and 
$\mathcal{M}$-independent.

Theorem 2.3 Let $\left(D=(V+s, A), \mathcal{M}=\left(\partial_{s}(V), r\right)\right)$ be a matroid-rooted digraph, where $\mathcal{M}$ is a transversal matroid. There exists an $\mathcal{M}$-based packing of spanning s-arborescences in $D$ if and only if $D$ is $\mathcal{M}$-ac.

Our main result is that Conjecture 1.2 is false in its general form. Moreover, the following decision problem is NP-complete.

Problem 2.4 Given a matroid-rooted digraph $(D=(V+s, A), \mathcal{M})$, decide whether there exists an $\mathcal{M}$-based packing of spanning s-arborescences in $D$.

Theorem 2.5 There exist an acyclic digraph $D=(V+s, A)$ and a matroid $\mathcal{M}$ of rank three such that $(D, \mathcal{M})$ is a counterexample to Conjecture 1.2.

Theorem 2.6 Problem 2.4 is NP-complete even if $D=(V+s, A)$ is acyclic and $\mathcal{M}$ is a linear matroid of rank three with a given linear representation.

Key ideas. We present the main ideas of the proofs below. The full proofs can be found in [4]. We note that our proofs for the positive cases also imply algorithms to find the packing, however, when the matroid is transversal, this algorithm needs a bipartite graph representing this matroid instead of its rank oracle.

For Theorems 2.1 and 2.2, we apply the proof of [2] to Conjecture 1.2. The shifting (of $(D, \mathcal{M}))$ along a pair $(u v, x)$ of an arc $u v$ and an element $x$ of $\mathcal{M}$ such that its corresponding root arc enters $u$ is a new instance $\left(D^{\prime}, \mathcal{M}^{\prime}\right)$ obtained from $(D, \mathcal{M})$ by removing $u v$ and inserting a new root arc sv such that $s v$ is a parallel element to $x$ in the underlying matroid. It is proved in [2] that if there is no packing formed by the root arcs there exists a pair $(e, x)$ of an arc and a matroid element such that the shifting $\left(D^{\prime}, \mathcal{M}^{\prime}\right)$ along $(e, x)$ is $\mathcal{M}^{\prime}$ independent and $\mathcal{M}^{\prime}$-ac. In the same manner, by induction, one gets an $\mathcal{M}^{\prime}$ based packing $\mathcal{T}$ of spanning $s$-arborescences in $D^{\prime}$. Our goal is to construct a feasible packing in $D$ based on $\mathcal{T}$. Let $T \in \mathcal{T}$ be an arborescence that covers the new root arc $f$ of $D^{\prime}$. If $T$ also contains $x$, then $(\mathcal{T}-\{T\}) \cup\{T-f+e\}$ is a feasible packing in $D$, and we are done. The difficult case is when $T$ does not contain $x$.

Matroid of rank at most 2. The proof of [2] fails only when the packing consists of two arborescences $T_{1}$ and $T_{2}$ (thus the rank of $\mathcal{M}^{\prime}$ is 2), w.l.o.g. assume $x \in T_{1}$ and $f \in T_{2}$. Let $V_{f} \subseteq V$ be the set of vertices which is reachable from $s$ in $T_{2}$ by a path starting with the arc $f$ or an arc parallel to $f$ in $\mathcal{M}$. Let $\left\{T_{1}^{*}, T_{2}^{*}\right\}$ be the packing that arises from $\left\{T_{1}, T_{2}\right\}$ by exchanging the arcs $T_{1}(v)$ and $T_{2}(v)$ for every vertex $v$ in $V_{f}$. Then we can prove that $\left\{T_{1}^{*}, T_{2}^{*}\right\}$ is a feasible packing in $D^{\prime}$ where $x$ and $f$ are in $T_{1}^{*}$. Thus we are in a case 
already treated.

Graphic matroids. Let $G=(\{0,1, \ldots, k\}, E)$ be a connected undirected graph representing $\mathcal{M}$, so the edges of $G$ corresponds to the root arcs of $D$. The idea is to require for the packing that each root arc may belong to $T_{i}$ only if its corresponding edge is incident to $i$ in $G$. This condition gives an extra property for the packing obtained by induction, based on which we show how to extend the packing keeping to be $\mathcal{M}$-based.

Transversal matroids. Let $G=(S, T ; E)$ be a bipartite graph representing $\mathcal{M}$ where $S$ corresponds to the set of root arcs of $D$ and $T=\{1, \ldots, k\}$. The plan is to replace the matroid-based condition by the following new condition: a root arc may belong to $T_{i}$ only if its corresponding vertex is connected to $i$ in $G$. It is much easier to deal with this condition, and the key observation is that if a packing of arborescences satisfies this new condition then any set of $k$ root arcs belonging to different arborescences of the packing forms a base of $\mathcal{M}$. Thus the packing is automatically $\mathcal{M}$-based. Let $D^{*}=\left(V^{*}, A^{*}\right)$ be the digraph that arises from $D$ by splitting $s$ into $|S|$ new vertices of out-degree one and define the sets $R_{i}(i=1, \ldots, k)$ to be the set of the tails of the image of root edges in $D^{*}$ which are connected to $i$ in $G$. Using these ideas the proof follows easily by a result of Katoh, Kamiyama and Takizawa [6].

Counterexample and NP-completeness. One of the simplest non-graphic and non-transversal matroids is the Fano matroid. A simple proof shows that Conjecture 1.2 is true for the Fano matroid when the digraph is acyclic. However, it turns out that Conjecture 1.2 is false when we allow to extend the Fano matroid by parallel elements. The symmetry of the Fano matroid is widely explored in the proof, and also its principal property is important that every pair of its elements is contained in a dependent set of cardinality 3, i.e. in a line of the Fano plane. We construct our acyclic digraph step by step by adding sink vertices of in-degree 3 . This construction ensures not only the existence of the required $\mathcal{M}$-based path packings but also that every $\mathcal{M}$-based arborescence packing is an extension of the previous instance. We design each construction step so that possible extensions are restricted, see Fig. 2.

Acknowledgments. This research was supported by the Project RIME of the laboratory G-SCOP. and by the Hausdorff Trimester Program on Combinatorial Optimization of the Hausdorff Research Institute, the University of Bonn, where this work was partially carried out. The second author was supported by the Hungarian Scientific Research Fund - OTKA, K109240, and by the MTA-ELTE Egerváry Research Group. The fourth author was supported by JSPS Postdoctoral Fellowships for Research Abroad and JSPS Grant-inAid for Scientific Research (B) 25280004. 


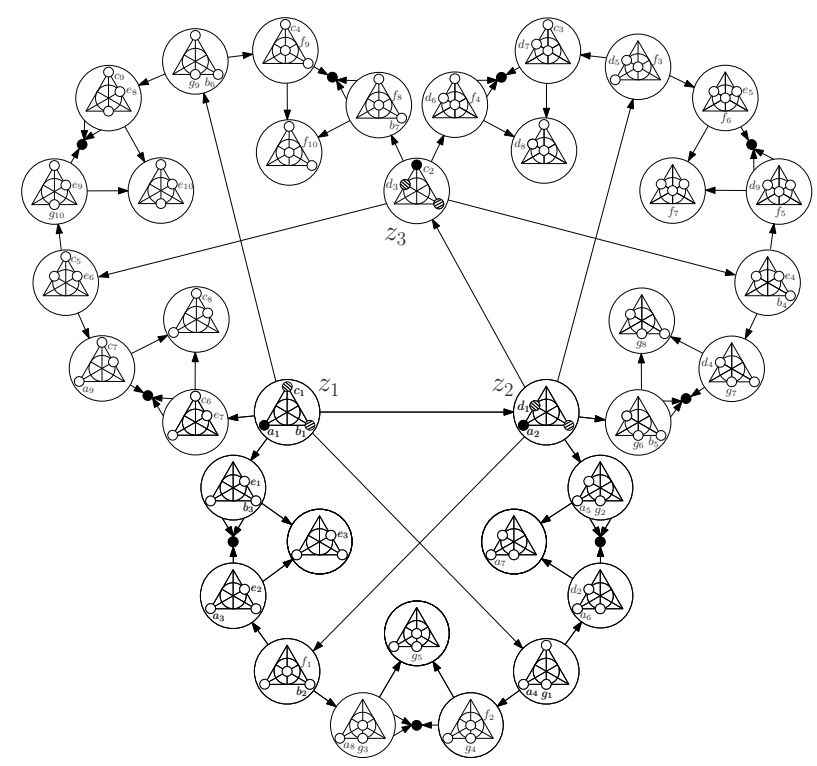

Fig. 1. A counterexample to Conjecture 1.2 without the root and the root arcs that are such that each non-root vertex has in-degree 3 . It is ensured by the construction of the matroid that, in any $\mathcal{M}$-based packing of spanning arborescences, the corresponding base to each "bigger" vertices is a parallel copy of the drawn one. Each leaf of the trefoil ensures that in the corresponding bases of $z_{i}$ and $z_{i+1}(i=1,2,3)$ the two parallel pairs of elements are in the same arborescence, a contradiction.

\section{References}

[1] Bérczi, K., T. Király, and Y. Kobayashi, Covering intersecting bi-set families under matroid constraints, SIAM J. Discrete Math., 30(2016), 1758-1774.

[2] Durand de Gevigney, O., V.H. Nguyen, and Z. Szigeti, Matroid-based packing of arborescences, SIAM J. Discrete Math., 27(2013), 567-574.

[3] Edmonds, J., Edge-disjoint branchings, in: B. Rustin (ed.) Combinatorial Algorithms, Academic Press, New York, 91-96 (1973)

[4] Fortier, Q., Cs. Király, Z. Szigeti, and S. Tanigawa, "On packing spanning arborescences with matroid constraint", EGRES Technical Report No. TR2016-18 (2016) URL: http://www.cs.elte.hu/egres.

[5] Frank, A., Personal communication (2013)

[6] Kamiyama, N., N. Katoh, and A. Takizawa, Arc-disjoint in-trees in directed graphs, Combinatorica, 29(2009), 197-214.

[7] Oxley, J.G. "Matroid theory", 2nd ed., Oxford University Press (2011) 